\title{
Hemodynamic and Biochemical Benefits of the Objective Measurement of Fluid Status in Hemodialysis Patients
}

\author{
Sejoong Kim, ${ }^{1,2}$ Jiyoon Sung, ${ }^{3}$ Eun Sook Jung, ${ }^{2}$ Hayne Cho Park, ${ }^{2,4}$ Hajeong Lee, ${ }^{2,4}$ \\ Ho Jun Chin, ${ }^{1,2}$ Dong Ki Kim, ${ }^{2,4}$ Yon Su Kim, ${ }^{2,4}$ Jin Suk Han ${ }^{2,4}$ and \\ Kwon Wook Joo ${ }^{2,4}$ \\ ${ }^{1}$ Department of Internal Medicine, Seoul National University Bundang Hospital, Seongnam, Republic of Korea \\ ${ }^{2}$ Department of Internal Medicine, Seoul National University College of Medicine, Seoul, Republic of Korea \\ ${ }^{3}$ Department of Internal Medicine, Gachon University of Medicine and Science, Seoul, Republic of Korea \\ ${ }^{4}$ Department of Internal Medicine, Seoul National University Hospital, Seoul, Republic of Korea
}

\begin{abstract}
Subtle fluid imbalance can cause poor clinical outcomes among hemodialysis patients. However, the traditional subjective assessment of fluid status may be inadequate. We evaluated whether the objective measurement and optimization of fluid status could be beneficial for hemodynamic and biochemical parameters in hemodialysis patients. We enrolled 120 hemodialysis patients, who were clinically euvolemic for at least 3 months. Based on the results of a body composition monitor, we divided the patients into the following two groups: the hyperhydrated group (post hemodialysis fluid overload $\geq 1.1 \mathrm{~L}$ ) and the dehydrated group (post hemodialysis fluid overload $<-1.1 \mathrm{~L}$ ). We reduced the patient's body weight in the hyperhydrated group and raised the body weight in the dehydrated group towards normohydration $(-1.1 \mathrm{~L}$ $\leq$ fluid overload $<1.1 \mathrm{~L}$ ) for 16 weeks. Forty-four of 120 patients were in the hyperhydrated group, and 18 of 120 patients in the dehydrated group. After 16 weeks, systolic blood pressure and pulse pressure decreased in the hyperhydrated group, while there was no increase in blood pressure in the dehydrated group after the intervention. Serum levels of monocyte chemotactic protein-1, an inflammatory marker, gradually decreased in the hyperhydrated group, and serum adiponectin levels, an anti-atherogenic biomarker, increased in the two groups. We found that hyperhydrated patients contributed over $1 / 3$ of the participants despite enrolling clinically euvolemic patients and that body composition monitor-guided optimization of body fluid status may lead to improvement of inflammatory markers and anti-atherogenic adipokines as well as hemodynamic parameters in hemodialysis patients.
\end{abstract}

Keywords: adipokines; blood pressure; body composition; hemodialysis; inflammation

Tohoku J. Exp. Med., 2012 Oct, 228 (2), 125-133. $\quad$ (C) 2012 Tohoku University Medical Press

In hemodialysis (HD) patients, chronic fluid overload can cause left ventricular hypertrophy (Jaeger and Mehta 1999), whereas dehydration is linked to the development of intradialytic adverse events such as hypotension (Passauer et al. 1998). Therefore, subtle fluid imbalance can lead to poor clinical outcomes among these patients. The kidney is the primary organ that controls volume status in the body. In HD patients, who have lost their renal function and depend on dialysis machines for most of their renal functions, adequate volume assessment is very important for maintaining normohydration. However, the traditional assessment of fluid status in HD patients depends on physicians' subjective evaluation, which means that that assessment may depend on physicians' experiences or be relatively insensitive.

Recently, a body composition monitor (BCM), a type of multi-frequency bioimpedance analysis device, was used for the objective assessment of fluid status in dialysis patients (Tattersall 2009). A large observational study has demonstrated that hyperhydration measured by the BCM is associated with higher mortality in HD patients (Wizemann et al. 2009). Another small longitudinal study found that the ratio of intracellular water to body weight (reflecting malnutrition) may have a negative effect on cardiac biomarkers and that the ratio of extracellular water to body weight (reflecting hyperhydration) may have a positive effect on those markers. Moreover, they showed that cardiac biomarkers were related to inflammation (Jacobs et al. 2010). In a recent interventional study, BCM-guided volume reduction led to better control of hypertension in hyperhydrated patients, but no biomarkers were measured (Machek et al. 2010). In contrast, Bednarek-Skublewska et

Received March 30, 2012; accepted August 23, 2012. Published online September 13, 2012; doi: 10.1620/tjem.228.125.

Correspondence: Kwon Wook Joo, M.D., Ph.D., Department of Internal Medicine, Seoul National University Hospital, 101 Daehakro, Jongro-gu, Seoul 110-744, Korea.

e-mail: junephro@paran.com 
al. (2010) showed that levels of cardiac biomarker were unrelated to the volumes of the body fluid compartments in HD patients; controversy remains regarding the relationship between biomarkers and body composition, and these studies were based on the observation studies (BednarekSkublewska et al. 2010; Jacobs et al. 2010), instead of on interventional studies.

Therefore, we evaluated the BCM-guided volume assessment in clinically normohydrated HD patients and the effect of BCM-guided volume control on changes in clinical and chemical biomarkers for 16 weeks in these patients.

\section{Subjects and Methods}

Study design

We enrolled 120 patients who received HD for more than 6 months at 3 major dialysis centers (Gachon University Gil Hospital, Seoul National University Hospital, and Seoul National University Bundang Hospital). All of the patients' dry weights were stable, and they were clinically normohydrated for at least 3 months. Inclusion criteria were as follows: age 18 years old or greater, no change in dialysate composition, and less than a $5 \%$ change in dry weight within 3 months. Exclusion criteria were as follows: any diagnosed acute or chronic inflammatory state within 3 months, hospitalizationrelated dialysis within 3 months, diseases that produce local fluid accumulation and edema, active malignancy, currently taking diuretics, currently taking any medication with the potential to influence body composition, malnutrition, pregnancy, insertion of a cardiac pacemaker or defibrillator, or the amputation of any extremity. All of the patients provided informed consent, and the study was approved by the institutional review boards of each hospital.

\section{BCM measurement}

A BCM (Fresenius Medical Care, Bad Homburg, Germany) was used to determine the extracellular and total body water. Fluid overload was calculated using a physiological BCM model that is based on tissue properties (Chamney et al. 2007). Patients rested for 5 minutes before performing the BCM measurement, and electrodes were fixed to the hand and foot on one side of the body in a supine position. All of the nurses in the centers were trained to use the BCM. If any erroneous measurement was detected by the BCM on the basis of a measurement quality indicator, the nurse repeated the measurement.

\section{Study protocol}

This study was a multicenter 16-week interventional trial. When the measured post-HD fluid overload ranged between $-1.1 \mathrm{~L}$ and $1.1 \mathrm{~L}$, the fluid status was considered to be normohydration (Passauer et al. 2010). Based on the amount of fluid overload, we divided the patients into the following two groups: the hyperhydrated group (fluid overload $\geq 1.1 \mathrm{~L}$ ) and the dehydrated group (fluid overload $<-1.1 \mathrm{~L}$ ). Dry weight was calculated as follows: dry weight $=$ (post-HD body weight) - (post-HD fluid overload). We restricted food intake during BCM measurement. We optimized body weight towards each patient's dry weight by adjustment of the amount of ultrafiltration for the first 8 weeks, and we maintained each patient's dry weight for the subsequent 8 weeks. During the intervention in the first 8 weeks, post-dialytic weight was adjusted gradually in steps of $<0.5 \mathrm{~kg} /$ week, and the antihypertensive drugs were adjusted in parallel with changes in the fluid status.
We collected clinical data including hemoglobin, blood urea nitrogen, creatinine, albumin, cholesterol, glucose, C-reactive protein, calcium, phosphorus, parathyroid hormone, and electrolytes. We measured BCM before and 30 minutes after HD sessions every 4 weeks and extracellular water, intracellular water, estimate of fluid overload, lean tissue index, fat tissue index, and fat content were recorded.

All parameters were measured at the midweek dialysis session. Every 8 weeks, the acquired serum samples were centrifuged and the supernatants were collected and immediately frozen at $-70^{\circ} \mathrm{C}$ until subsequent analysis. All of the samples were run in duplicate to measure biochemical parameters. Leptin, adiponectin, and resistin levels were determined using Bio-Plex Pro human diabetes assays (Bio-Rad Laboratories, Hercules, CA, USA), with intra-assay coefficients of variation (CVs) of 5.38\%. Serum interleukin-6 (IL6) and monocyte chemotactic protein-1 (MCP1) concentrations were measured using the Bio-Plex multiplex cytokine assay (Bio-Rad Laboratories), with intra-assay CVs of $6.73 \%$. Serum lipopolysaccharide quantification for the measurement of endotoxin was performed using a Limulus Amebocyte assay (Cambrex, Verviers, Belgium), with an intra-assay CVs of $4.95 \%$.

\section{Statistical analysis}

Continuous variables are expressed as the means \pm S.D., whereas categorical variables are presented as absolute values and percentages. Differences between groups were tested with one-way analysis of variance (ANOVA) for continuous variables and with the chisquare test for categorical variables. Intra-group differences for continuous variables were assessed using the Wilcoxon signed ranks test and repeated measures ANOVA for paired samples. Linear regression analysis was performed according to Spearman's test. Due to their skewed distribution, the values were log transformed.

The SPSS (SPSS version 12.0, Chicago, IL, USA) package was used for statistical analysis. A $P$-value of $<0.05$ was considered significant. All probabilities were two-tailed.

\section{Results \\ Baseline characteristics and clinical course}

The demographic features and BCM parameters of each study group are shown in Table 1. The mean age was 55.6 years, and half of the patients had diabetes mellitus. Dialysis vintage was approximately 5 years, and the prevalence of anuria was $83 \%$. Of the patients, $36.6 \%(44 / 120)$ were in the hyperhydrated group, and $15.0 \%(18 / 120)$ were in the dehydrated group. Fig. 1 presents the trends throughout the study. In 44 hyperhydrated patients, 7 patients did not complete the study due to the following reasons: transfer to another hospital, 3; hospitalization, 2; and withdrawal of informed consent, 2 . In 18 dehydrated patients, 2 patients did not complete the study due to transplantation (1) and hospitalization (1). Fifty-three of 62 patients $(85.5 \%)$ completed the study and were included in the data analysis. The percentage of angiotensin-converting enzyme inhibitor or angiotensin II receptor blocker usage was 10\% in the dehydrated group, and $73.3 \%$ in the hyperhydrated group. All enrolled patients underwent three dialysis sessions per week. 
Table 1. Baseline characteristics of the patients.

\begin{tabular}{|c|c|c|c|c|c|}
\hline & Total & Dehydration & Normohydration & Hyperhydration & $P$ \\
\hline $\mathrm{N}$ & 120 & 18 & 58 & 44 & \\
\hline Post-hemodialysis fluid overload (L) & $0.89 \pm 2.15$ & $-1.90 \pm 0.61$ & $0 \pm 0.58$ & $2.99 \pm 1.69$ & $<0.001$ \\
\hline Male (\%) & $67(55.4)$ & $8(42.1)$ & $30(52.6)$ & $28(63.6)$ & 0.255 \\
\hline Age (year) & $56.4 \pm 13.2$ & $53.3 \pm 14.3$ & $55.9 \pm 14.1$ & $58.4 \pm 11.3$ & 0.360 \\
\hline Cause of end stage renal disease (\%) & & & & & 0.106 \\
\hline Diabetes & $55(45.5)$ & $4(21.1)$ & $27(47.4)$ & $23(52.3)$ & \\
\hline Hypertension & $20(16.5)$ & $3(15.8)$ & $11(19.3)$ & $6(13.6)$ & \\
\hline Glomerulonephritis & $16(13.2)$ & $6(31.6)$ & $7(12.3)$ & $3(6.82)$ & \\
\hline Others & $30(24.8)$ & $6(31.6)$ & $12(21.1)$ & $12(27.3)$ & \\
\hline Dialysis vintage (year) & $4.97 \pm 4.73$ & $5.98 \pm 5.64$ & $4.1 \pm 3$ & $5.71 \pm 5.95$ & 0.146 \\
\hline Height (cm) & $163 \pm 8.90$ & $160 \pm 8.69$ & $163 \pm 8.27$ & $164 \pm 9.67$ & 0.274 \\
\hline Body weight (kg) & $57.2 \pm 9.43$ & $55.1 \pm 8.32$ & $58.6 \pm 10.8$ & $56.4 \pm 7.97$ & 0.365 \\
\hline Urine output (mL/day) & $120 \pm 216$ & $106 \pm 189$ & $122 \pm 234$ & $123 \pm 207$ & 0.956 \\
\hline Anuria $(\%)$ & $100(82.6)$ & $15(79.0)$ & $48(84.2)$ & $36(81.8)$ & 0.863 \\
\hline
\end{tabular}

Values are mean \pm S.D.

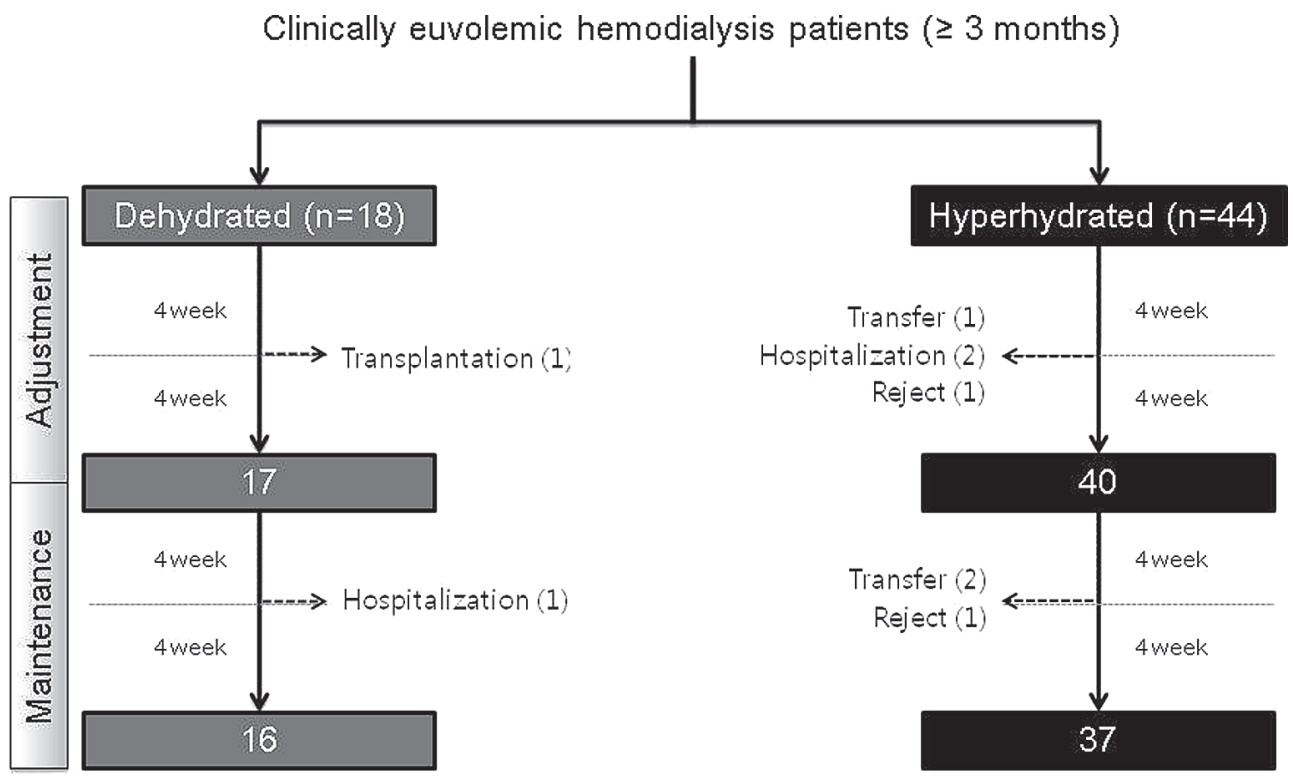

Fig. 1. Flow diagram of patients enrolled in the study.

\section{Change in the parameters}

Changes in post-HD fluid overload of both the dehydrated and hyperhydrated groups after the 16-week intervention are shown in Fig. 2. In the hyperhydrated group, postHD fluid overload was significantly decreased after the 8-week intervention $(2.99 \pm 1.69 \mathrm{~L}$ at week $0,1.21 \pm 1.43 \mathrm{~L}$ at week 8 , and $0.74 \pm 2.35 \mathrm{~L}$ at week 16 ). In the dehydrated group, post-HD fluid overload was increased, but there was no significant difference $(-1.90 \pm 0.61 \mathrm{~L}$ at week $0,-1.41 \pm$ $1.13 \mathrm{~L}$ at week 8 , and $-1.29 \pm 1.08 \mathrm{~L}$ at week 16 ). Systolic blood pressure and pulse pressure after the HD session were significantly decreased after the 16-week intervention in hyperhydrated patients (Table 2). The number of anti- hypertensive drugs was also decreased in the hyperhydrated group. In dehydrated patients, there was no increase in blood pressure parameters after the 16-week intervention.

The fat tissue index at the post-HD session was markedly increased in the dehydrated patients. This result suggests that fat content may be the main contributor to an increase in body weight. In hyperhydrated patients, extracellular water at pre- and post-HD sessions and intracellular water at post-HD sessions were decreased after the intervention, while fat tissue index and fat content at the time of the post-HD session were increased after the intervention (Table 3).

In both groups, serum MCP1 levels decreased gradu- 


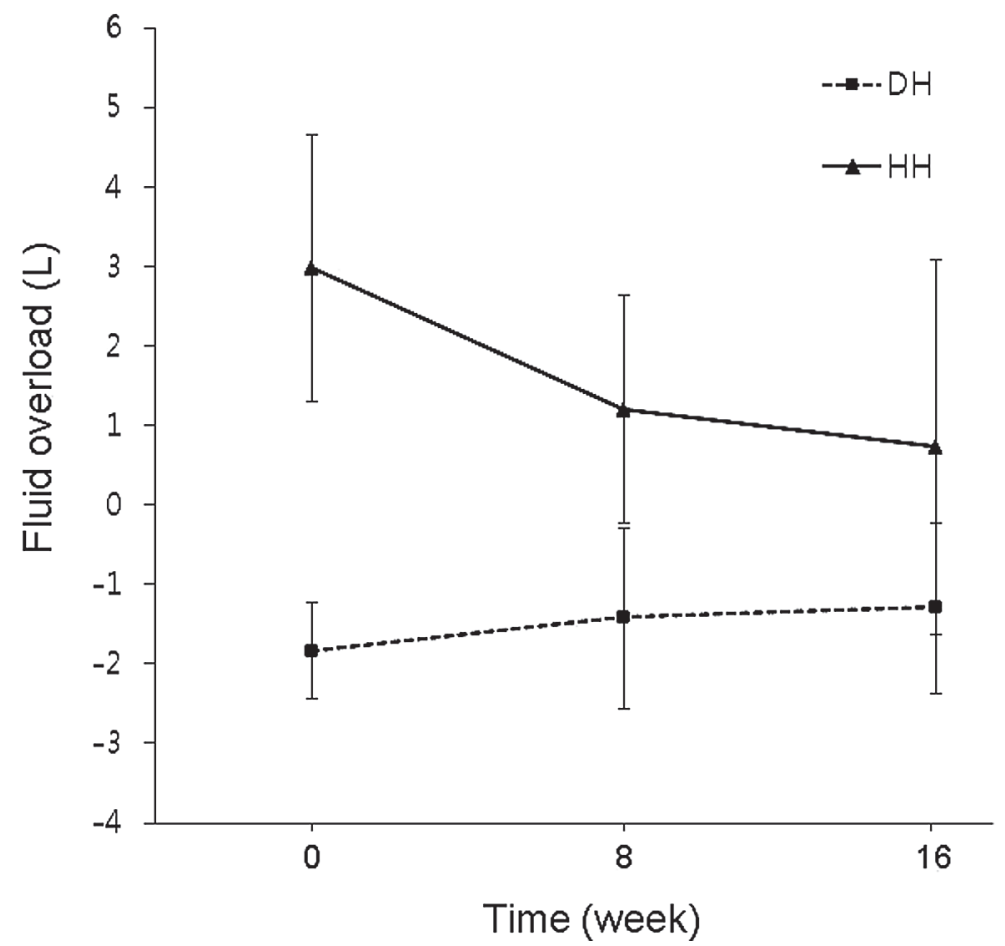

Fig. 2. Changes in post-hemodialysis fluid overload.

Post-hemodialysis fluid overload was decreased in the hyperhydrated group $(\mathrm{HH})$ and increased in the dehydrated group (DH) after 16 weeks of BCM-guided intervention ( $n=44$ at week 0, 40 at week 8, and 37 at week 16 in $\mathrm{HH} ; n=18$ at week 0,17 at week 8 , and 16 at week 16 in $\mathrm{DH})$.

Table 2. Changes in haemodynamic parameters in dehydrated and hyperhydrated patients.

\begin{tabular}{|c|c|c|c|c|}
\hline Parameters & Week 0 & Week 8 & Week 16 & ${ }^{*} P$ \\
\hline \multicolumn{5}{|l|}{ Dehydration } \\
\hline Systolic $\mathrm{BP}_{\text {pre }}(\mathrm{mm} \mathrm{Hg})$ & $128 \pm 15.5$ & $132 \pm 23.1$ & $130 \pm 22.3$ & 0.638 \\
\hline Diastolic $\mathrm{BP}_{\mathrm{pre}}(\mathrm{mm} \mathrm{Hg})$ & $72.9 \pm 13.4$ & $74.0 \pm 17.9$ & $70.7 \pm 14.9$ & 0.272 \\
\hline Pulse Pressure $_{\text {pre }}(\mathrm{mm} \mathrm{Hg})$ & $55.8 \pm 11.8$ & $58.6 \pm 15.4$ & $59.7 \pm 14.0$ & 0.778 \\
\hline Hypotensive event (per week) & $0.53 \pm 1.01$ & $0.27 \pm 0.8$ & $0.19 \pm 0.40$ & 0.300 \\
\hline No. of anti-hypertensive drugs & $1.29 \pm 1.45$ & $1.2 \pm 1.42$ & $1.33 \pm 1.5$ & 0.666 \\
\hline \multicolumn{5}{|l|}{ Hyperhydration } \\
\hline Systolic $\mathrm{BP}_{\text {pre }}(\mathrm{mm} \mathrm{Hg})$ & $150 \pm 16.2$ & $146 \pm 18.7$ & $143 \pm 21.0^{\dagger}$ & 0.025 \\
\hline Diastolic $\mathrm{BP}_{\text {pre }}(\mathrm{mm} \mathrm{Hg})$ & $71.4 \pm 11.0$ & $71.4 \pm 13.0$ & $70.7 \pm 11.2$ & 0.611 \\
\hline Pulse Pressure $_{\text {pre }}(\mathrm{mm} \mathrm{Hg})$ & $78.6 \pm 13.4$ & $72.5 \pm 21.4$ & $71.7 \pm 20.4^{\dagger}$ & 0.022 \\
\hline Hypotensive event (per week) & $0.18 \pm 0.64$ & $0.21 \pm 0.52$ & $0.28 \pm 0.74$ & 0.664 \\
\hline No. of anti-hypertensive drugs & $4.38 \pm 2.67$ & $4.41 \pm 2.59$ & $4.05 \pm 2.53^{\dagger+}$ & 0.182 \\
\hline
\end{tabular}

Values are mean \pm S.D.

BP, blood pressure; pre, pre-hemodialysis; post, post-hemodialysis.

$* P<0.05$ using repeated-measures analysis of variance.

${ }^{\dagger} P<0.05$ vs. week 0 , and $₫ P<0.05$ vs. week 8 using the Wilcoxon signed ranks test.

ally during the 16-week period (Table 4). Although serum levels of IL6 were significantly decreased after the 8-week intervention period, these levels fluctuated in both groups during the subsequent 8 -week period. In the hyperhydrated patients, endotoxin levels were also decreased during the first 8-week period, although these effects were not sustained during the subsequent 8 -week period. In the dehydrated group, serum adiponectin levels gradually increased during the 16-week intervention, while serum leptin and resistin levels were not changed. In the hyperhydrated 
Table 3. Changes in parameters of body composition in the dehydrated and hyperhydrated patients.

\begin{tabular}{|c|c|c|c|c|}
\hline & Week 0 & Week 8 & Week 16 & $P$ \\
\hline \multicolumn{5}{|l|}{ Dehydration } \\
\hline $\mathrm{ECW}_{\text {pre }}(\mathrm{L})$ & $13.5 \pm 2.02$ & $13.5 \pm 2.08$ & $13.6 \pm 2.10$ & 0.497 \\
\hline $\mathrm{ICW}_{\text {pre }}(\mathrm{L})$ & $16.2 \pm 3.63$ & $15.9 \pm 2.71$ & $15.5 \pm 2.60$ & 0.305 \\
\hline $\mathrm{ECW} / \mathrm{ICW}_{\text {pre }}$ & $0.85 \pm 0.11$ & $0.86 \pm 0.10$ & $0.88 \pm 0.06$ & 0.141 \\
\hline Lean tissue index $x_{\text {pre }}(\mathrm{L})$ & $13.2 \pm 2.74$ & $12.9 \pm 1.98$ & $12.4 \pm 1.73$ & 0.222 \\
\hline Fat tissue index $x_{\text {pre }}(\mathrm{L})$ & $9.06 \pm 4.04$ & $9.61 \pm 3.44$ & $9.86 \pm 3.32$ & 0.178 \\
\hline Fat content $t_{\text {pre }}(\%)$ & $28.5 \pm 10.4$ & $30.2 \pm 8.01$ & $31.1 \pm 7.94$ & 0.167 \\
\hline Ultrafiltration (L/session) & $2.47 \pm 0.69$ & $2.05 \pm 0.84$ & $2.27 \pm 0.61$ & 0.155 \\
\hline $\mathrm{ECW}_{\text {post }}(\mathrm{L})$ & $11.6 \pm 1.90$ & $11.6 \pm 1.82$ & $11.5 \pm 1.8$ & 0.810 \\
\hline $\mathrm{ICW}_{\text {post }}(\mathrm{L})$ & $16.6 \pm 3.28$ & $16.1 \pm 2.85$ & $15.8 \pm 2.69$ & 0.059 \\
\hline $\mathrm{ECW} / \mathrm{ICW}_{\text {post }}$ & $0.71 \pm 0.09$ & $0.73 \pm 0.09$ & $0.73 \pm 0.07$ & 0.192 \\
\hline Lean tissue index ${ }_{\text {post }}(\mathrm{L})$ & $13.4 \pm 2.27$ & $13.1 \pm 2.11$ & $12.7 \pm 1.77$ & 0.076 \\
\hline Fat tissue index $x_{\text {post }}(\mathrm{L})$ & $8.89 \pm 3.87$ & $9.35 \pm 3.55^{\dagger}$ & $9.63 \pm 3.55^{\dagger}$ & 0.009 \\
\hline Fat content $\mathrm{post}(\%)$ & $28.9 \pm 9.78$ & $30.4 \pm 8.93^{\dagger}$ & $31.3 \pm 8.83^{\dagger}$ & 0.014 \\
\hline \multicolumn{5}{|l|}{ Hyperhydration } \\
\hline $\mathrm{ECW}_{\text {pre }}(\mathrm{L})$ & $17.0 \pm 3.06$ & $15.5 \pm 2.44^{\dagger}$ & $15.4 \pm 2.63^{\dagger}$ & $<0.001$ \\
\hline $\mathrm{ICW}_{\text {pre }}(\mathrm{L})$ & $15.7 \pm 3.68$ & $15.9 \pm 3.22$ & $16.3 \pm 4.37$ & 0.422 \\
\hline $\mathrm{ECW} / \mathrm{ICW}_{\mathrm{pre}}(\mathrm{L})$ & $1.11 \pm 0.21$ & $0.99 \pm 0.14^{\dagger}$ & $0.99 \pm 0.19^{\dagger}$ & 0.001 \\
\hline Lean tissue index $x_{\text {pre }}(\mathrm{L})$ & $12.3 \pm 2.64$ & $12.5 \pm 2.26$ & $12.2 \pm 2.33^{\ddagger}$ & 0.384 \\
\hline Fat tissue index $x_{\text {pre }}(\mathrm{L})$ & $7.51 \pm 3.51$ & $7.59 \pm 3.53$ & $8.07 \pm 3.74^{\ddagger}$ & 0.217 \\
\hline Fat content $t_{\text {pre }}(\%)$ & $24.9 \pm 9.78$ & $25.3 \pm 9.38$ & $26.8 \pm 9.61^{\dagger t}$ & 0.149 \\
\hline Ultrafiltration (L/session) & $1.65 \pm 1.35$ & $1.84 \pm 1.02$ & $1.93 \pm 1.23$ & 0.512 \\
\hline $\mathrm{ECW}_{\text {post }}(\mathrm{L})$ & $15.8 \pm 2.73$ & $14.3 \pm 2.12^{\dagger}$ & $14.1 \pm 2.25^{\dagger}$ & $<0.001$ \\
\hline $\mathrm{ICW}_{\text {post }}(\mathrm{L})$ & $16.5 \pm 4.33$ & $15.8 \pm 5.55^{\dagger}$ & $14.8 \pm 3.72^{\dagger+}$ & 0.018 \\
\hline $\mathrm{ECW} / \mathrm{ICW}_{\text {post }}(\mathrm{L})$ & $1.00 \pm 0.23$ & $0.96 \pm 0.19$ & $0.99 \pm 0.19$ & 0.316 \\
\hline Lean tissue index $x_{\text {post }}(\mathrm{L})$ & $12.7 \pm 2.7$ & $11.7 \pm 2.53^{\dagger}$ & $11.3 \pm 2.61^{\dagger+}$ & $<0.001$ \\
\hline Fat tissue index $x_{\text {post }}(\mathrm{L})$ & $7.20 \pm 2.81$ & $7.73 \pm 3.36^{\dagger}$ & $8.08 \pm 3.51^{\dagger+}$ & 0.001 \\
\hline Fat content $\mathrm{post}(\%)$ & $24.8 \pm 8.12$ & $27.2 \pm 10.1^{\dagger}$ & $28.5 \pm 10.3^{\dagger t}$ & $<0.001$ \\
\hline
\end{tabular}

Values are mean \pm S.D.

ECW, extracellular water; ICW, intracellular water; pre, pre-hemodialysis; post, post-hemodialysis.

Table 4. Changes in cytokines in the dehydrated and hyperhydrated patients.

\begin{tabular}{|c|c|c|c|c|}
\hline Parameters & Week 0 & Week 8 & Week 16 & ${ }^{*} P$ \\
\hline \multicolumn{5}{|l|}{ Dehydration } \\
\hline Interleukin-6 $(\ln [\mathrm{pg} / \mathrm{mL}])$ & $1.29 \pm 2.58$ & $0.04 \pm 1.15 \dagger$ & $1.29 \pm 0.76 \%$ & 0.100 \\
\hline MCP1 (ln[pg/mL]) & $5.89 \pm 1.53$ & $5.33 \pm 0.48 \dagger$ & $5.37 \pm 0.50$ & 0.095 \\
\hline Leptin $(\ln [\mathrm{pg} / \mathrm{mL}])$ & $10.3 \pm 1.78$ & $9.99 \pm 1.65$ & $10.1 \pm 1.71$ & 0.817 \\
\hline Adiponectin $(\ln [\mathrm{pg} / \mathrm{mL}])$ & $14.0 \pm 0.14$ & $15.8 \pm 0.31 \dagger$ & $16.1 \pm 0.40+t$ & $<0.001$ \\
\hline Resistin $(\ln [\mathrm{pg} / \mathrm{mL}])$ & $10.2 \pm 0.49$ & $10.1 \pm 0.46$ & $10.2 \pm 0.52$ & 0.661 \\
\hline Endotoxin $(\ln [\mathrm{EU} / \mathrm{mL}])$ & $1.05 \pm 0.50$ & $1.01 \pm 0.40$ & $1.07 \pm 0.30$ & 0.908 \\
\hline \multicolumn{5}{|l|}{ Hyperhydration } \\
\hline Interleukin-6 $(\ln [\mathrm{pg} / \mathrm{mL}])$ & $1.13 \pm 1.32$ & $0.04 \pm 1.16^{\dagger}$ & $1.23 \pm 0.55^{\ddagger}$ & $<0.001$ \\
\hline MCP1 $(\ln [\mathrm{pg} / \mathrm{mL}])$ & $5.64 \pm 0.77$ & $5.27 \pm 0.37^{\dagger}$ & $5.21 \pm 0.42^{\dagger}$ & 0.005 \\
\hline Leptin $(\ln [\mathrm{pg} / \mathrm{mL}])$ & $8.89 \pm 1.58$ & $8.37 \pm 1.55^{\dagger}$ & $8.66 \pm 1.60^{*}$ & 0.013 \\
\hline Adiponectin $(\ln [\mathrm{pg} / \mathrm{mL}])$ & $14.0 \pm 0.15$ & $15.8 \pm 0.33^{\dagger}$ & $16.3 \pm 0.44^{\dagger t}$ & $<0.001$ \\
\hline Resistin $(\ln [\mathrm{pg} / \mathrm{mL}])$ & $10.1 \pm 0.52$ & $10.0 \pm 0.50$ & $10.0 \pm 0.58$ & 0.782 \\
\hline Endotoxin $(\ln [\mathrm{EU} / \mathrm{mL}])$ & $0.96 \pm 0.43$ & $0.89 \pm 0.39^{\dagger}$ & $0.92 \pm 0.34$ & 0.232 \\
\hline
\end{tabular}

Values are mean \pm S.D

MCP1, monocyte chemotactic protein-1.

${ }^{*} P<0.05$ using by repeated-measures analysis of variance tests.

${ }^{\dagger} P<0.05$ vs. week 0 , and $₫ P<0.05$ vs. week 8 using the Wilcoxon signed ranks test. 
Table 5. Changes in biochemical parameters in dehydrated and hyperhydrated patients.

\begin{tabular}{|c|c|c|c|c|}
\hline & Week 0 & Week 8 & Week 16 & ${ }^{*} P$ \\
\hline \multicolumn{5}{|l|}{ Dehydration } \\
\hline Hemoglobin (g/dL) & $10.9 \pm 0.79$ & $10.6 \pm 0.98$ & $10.2 \pm 0.84$ & 0.111 \\
\hline Blood urea nitrogen $(\mathrm{mg} / \mathrm{dL})$ & $72.6 \pm 18.6$ & $68.8 \pm 14.5$ & $60.3 \pm 19.5^{\dagger}$ & 0.012 \\
\hline Creatinine (mg/dL) & $10.5 \pm 2.25$ & $10.5 \pm 2.10$ & $10.0 \pm 2.04^{\dagger+}$ & 0.097 \\
\hline Albumin (g/dL) & $3.89 \pm 0.32$ & $3.85 \pm 0.35$ & $3.85 \pm 0.40$ & 0.525 \\
\hline Cholesterol (mg/dL) & $162 \pm 25.6$ & $150 \pm 28.1$ & $155 \pm 20.12$ & 0.145 \\
\hline C-reactive protein $(\mathrm{mg} / \mathrm{dL})$ & $0.18 \pm 0.19$ & $0.34 \pm 0.44$ & $0.20 \pm 0.22$ & 0.757 \\
\hline Calcium (mg/dL) & $9.03 \pm 0.63$ & $8.99 \pm 0.71$ & $8.56 \pm 0.77$ & 0.187 \\
\hline Phosphorus (mg/dL) & $4.95 \pm 1.15$ & $4.35 \pm 1.53$ & $5.10 \pm 2.15$ & 0.888 \\
\hline Sodium $(\mathrm{mmol} / \mathrm{L})$ & $138 \pm 2.85$ & $138 \pm 2.41$ & $139 \pm 2.05 \dagger$ & $<0.001$ \\
\hline Potassium (mmol/L) & $5.09 \pm 0.72$ & $4.83 \pm 0.67$ & $5.03 \pm 0.78$ & 0.891 \\
\hline total $\mathrm{CO}_{2}(\mathrm{mmol} / \mathrm{L})$ & $19.9 \pm 3.57$ & $19.61 \pm 3.49$ & $17.1 \pm 4.84$ & 0.797 \\
\hline Parathyroid hormone (pg/dL) & $254 \pm 271$ & $294 \pm 293$ & $268 \pm 286$ & 0.445 \\
\hline $\mathrm{Kt} /$ Vurea & $1.68 \pm 0.17$ & $1.73 \pm 0.22$ & $1.79 \pm 0.17$ & 0.097 \\
\hline nPNA (g/kg/day) & $1.35 \pm 0.18$ & $1.31 \pm 0.18$ & $1.25 \pm 0.23$ & 0.289 \\
\hline \multicolumn{5}{|l|}{ Hyperhydration } \\
\hline Hemoglobin (g/dL) & $10.6 \pm 1.04$ & $10.7 \pm 1.23$ & $11.2 \pm 4.09$ & 0.641 \\
\hline Blood urea nitrogen (mg/dL) & $59.8 \pm 19.0$ & $64.3 \pm 15.0$ & $60.5 \pm 17.6$ & 0.240 \\
\hline Creatinine (mg/dL) & $8.83 \pm 2.34$ & $9.01 \pm 2.38$ & $8.82 \pm 2.66$ & 0.682 \\
\hline Albumin (g/dL) & $3.89 \pm 0.39$ & $3.89 \pm 0.5$ & $3.79 \pm 0.4^{\ddagger}$ & 0.204 \\
\hline Cholesterol (mg/dL) & $146 \pm 33.6$ & $143 \pm 28.8$ & $149 \pm 33.2$ & 0.475 \\
\hline C-reactive protein $(\mathrm{mg} / \mathrm{dL})$ & $0.16 \pm 0.26$ & $0.32 \pm 0.55$ & $0.54 \pm 0.82$ & 0.297 \\
\hline Calcium (mg/dL) & $8.65 \pm 0.7$ & $8.42 \pm 0.74^{\dagger}$ & $8.53 \pm 0.65^{\ddagger}$ & 0.016 \\
\hline Phosphorus (mg/dL) & $4.59 \pm 1.18$ & $4.97 \pm 1.41$ & $5.15 \pm 1.86$ & 0.284 \\
\hline Sodium (mmol/L) & $137 \pm 4.14$ & $138 \pm 4.48$ & $137 \pm 3.69$ & 0.397 \\
\hline Potassium (mmol/L) & $5.13 \pm 0.82$ & $5.15 \pm 0.79$ & $5.16 \pm 0.82$ & 0.926 \\
\hline total $\mathrm{CO}_{2}(\mathrm{mmol} / \mathrm{L})$ & $20.0 \pm 3.98$ & $19.0 \pm 3.94^{\dagger}$ & $18.8 \pm 3.68$ & 0.098 \\
\hline Parathyroid hormone (pg/dL) & $181 \pm 352$ & $190 \pm 349$ & $203 \pm 433$ & 0.262 \\
\hline $\mathrm{Kt} /$ Vurea & $1.48 \pm 0.18$ & $1.57 \pm 0.26$ & $1.50 \pm 0.27$ & 0.217 \\
\hline nPNA (g/kg/day) & $0.99 \pm 0.19$ & $1.13 \pm 0.21^{\dagger}$ & $1.01 \pm 0.17^{\ddagger}$ & 0.001 \\
\hline
\end{tabular}

Values are mean \pm S.D.

nPNA, normalized protein equivalent of nitrogen appearance.

group, similar to the dehydrated group, serum adiponectin levels gradually increased throughout the entire period. There was no significant change in other parameters in either group, including hemoglobin, albumin, and cholesterol (Table 5). The normalized protein equivalent of nitrogen appearance (nPNA) was slightly increased in the hyperhydrated group and unchanged in the dehydrated group.

As shown in Fig. 3, serum endotoxin levels were positively associated with serum MCP1 and IL6 $(r=0.556, P<$ 0.001 and $r=0.641, P<0.001$, respectively). However, serum endotoxin levels were negatively associated with serum adiponectin $(r=-0.326, P=0.005)$.

\section{Discussion}

We demonstrated that the objective assessment of fluid status can detect hyperhydrated or dehydrated state in subjectively euvolemic HD patients, and we also found that
BCM-guided optimization of fluid status may lead to the improvement of inflammatory markers and anti-atherogenic adipokines as well as hemodynamic parameters in HD patients.

Machek et al. (2010) showed that volume-guidance towards normohydration leads to better control of hypertension in hyperhydrated patients and less intradialytic adverse events in the dehydrated patients. This result is consistent with our data. However, the authors assessed the changes in hydration status retrospectively, which suggests that the data were not followed up on a regular basis and that they had no data on biochemical biomarkers. Our study design was prospective, and blood samples were collected every 8 weeks.

After the optimization of patients' volume status, systolic blood pressure, and pulse pressure in the hyperhydrated patients were significantly decreased without con- 
A

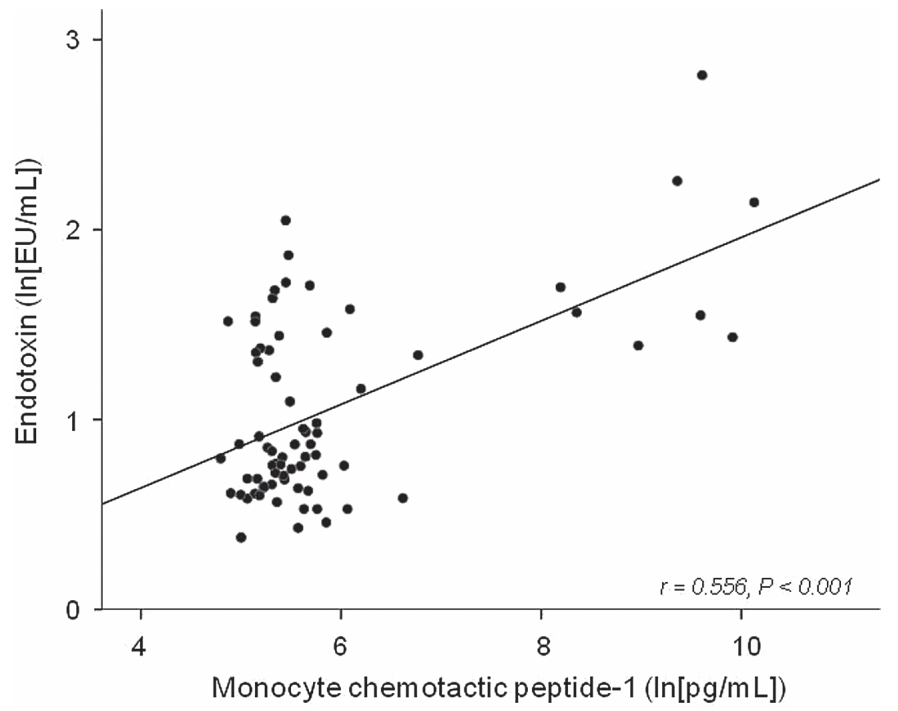

B

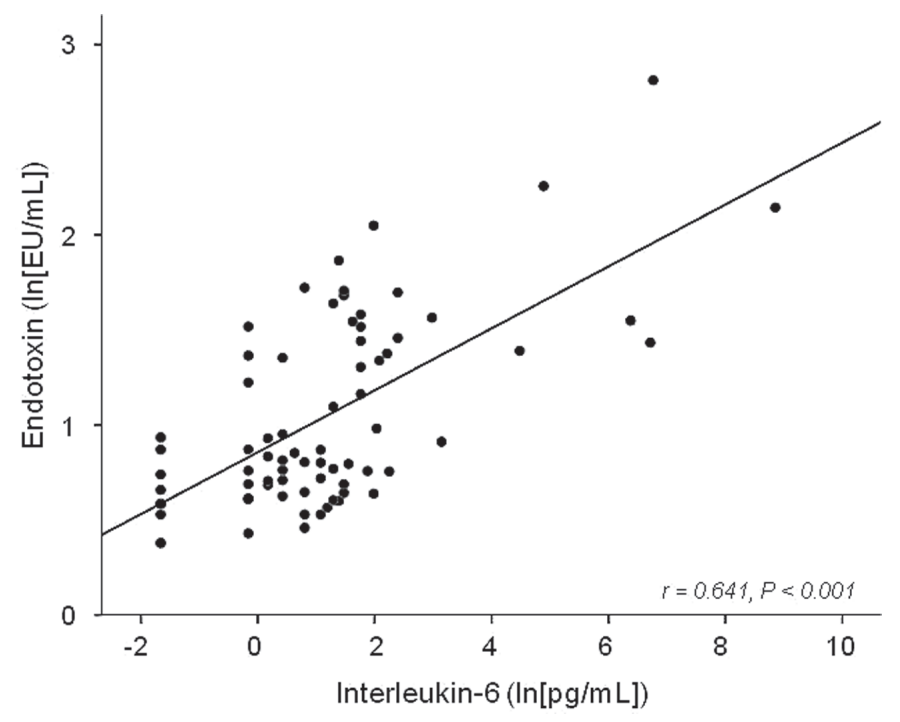

$\mathrm{C}$

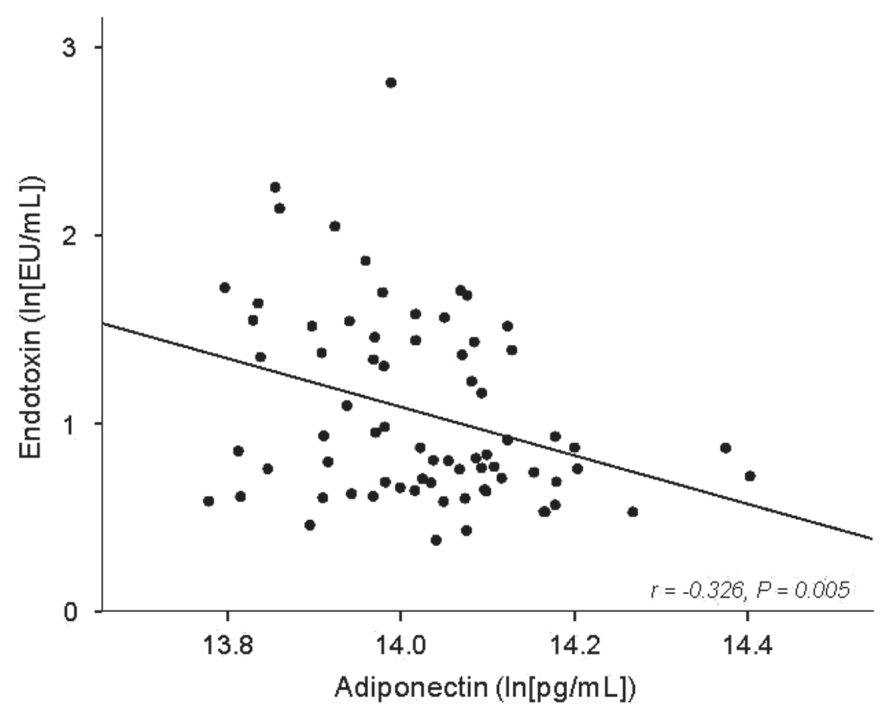

Fig. 3. Association between endotoxins and other cytokines in hemodialysis patients.

There were positive correlations between monocyte chemotactic peptide- 1 and endotoxin (A), and between interleukin-6 and endotoxin (B), while there was a negative correlation between adiponectin and endotoxin $(\mathrm{C})(n=73)$. Due to their skewed distribution, the values were log transformed. 
comitant increases in the dose of anti-hypertensive medication. In the dehydrated patients, there was a reduction in hypotensive episodes after the intervention, although this reduction was not statistically significant. This result may arise because most of the hyperhydrated patients reduced their fluid overload sequentially, while some of the dehydrated patients failed to reach normohydration after the 16-week intervention (Fig. 2).

$\mathrm{MCP} 1$, a monomeric polypeptide of 9 to $15 \mathrm{kDa}$, is the prototype of the $\mathrm{C}-\mathrm{C}$ chemokine $\mathrm{b}$ subfamily and also exhibits its most potent activity for monocytes (Ikeda et al. 2002). MCP1 is produced by various types of cells within the arterial wall, including vascular smooth muscle cells, endothelial cells and macrophages in response to wide ranges of stimuli. IL6 is a $26-\mathrm{kDa}$ cytokine that is produced by many different cells in the body (Ikeda et al. 2001). It has been well documented that elevated levels of acutephase proteins are associated with unfavorable short- and long-term prognoses in patients with end-stage renal disease (Pecoits-Filho et al. 2002). Hyperhydration is reported to be related to inflammation in patients with peritoneal dialysis (Vicente-Martinez et al. 2004). In this study, although IL6 levels were not changed, MCP1 levels in hyperhydrated patients were significantly decreased after the 8-week intervention; those changes were maintained for the subsequent 8 weeks.

In chronic kidney disease patients, endotoxin enters the circulation via bacterial translocation (the passage of intact bacteria and macromolecules such as endotoxin across the intestinal barrier) (Kotanko et al. 2006). In congestive heart failure, bowel edema and hypoperfusion may be the two main factors influencing bowel wall permeability (Krack et al. 2005). In HD patients, predialysis endotoxin levels could be associated with ultrafiltration volume or hypotensive events during the dialytic session (McIntyre et al. 2011). In this current study, endotoxemia was positively associated with IL6 and MCP1 levels and negatively associated with adioponectin levels. This result suggests that endotoxin may be a mediator of inflammation in the presence of hyperhydration; however, further study is needed to evaluate the association between volume control and inflammation.

The post-HD fat tissue index was increased in both dehydrated and hyperhydrated patients. In addition, nPNA, a nutritional marker in HD patients, was increased in the hyperhydrated patients after the 16-week intervention. Cheng et al. (2005) found that optimized fluid status was associated with an improvement in nutritional status in peritoneal dialysis patients. Fat has some favorable effects on storing energy and counteracts the inflammatory effects of circulating endotoxins (Mohamed-Ali et al. 1999). These data suggest that the correction of fluid imbalance may be closely related to the improvement of nutrition, especially in hyperhydrated patients.

Adipocytes synthesize and release adipokines, which have been linked to blood pressure control. Adiponectin is protective against hypertension through an endotheliumdependent mechanism, and plays an important role in the regulation of vascular tone (Ouchi et al. 2003). Shatat et al. (2009) showed that lower serum adiponectin levels were associated with higher blood pressures. In the hyperhydrated patients in the current study, serum adiponectin levels were increased and pre-HD systolic blood pressures and pulse pressures were decreased after a 16-week period. However, it is unclear whether the increased levels of adiponectin directly influence the change in blood pressure or is an epiphenomenon with increased fat tissue index.

This study had several limitations. There were no data on changes in biochemical parameters of normohydrated patients during the 16-week intervention period, which would be informative and important. This study is an interventional trial and not a randomized controlled study, which means that all the hyperhydrated patients were treated. Therefore, we focused on the changes according to the interventional period. Sixteen weeks may not be a sufficiently long period to evaluate hard clinical outcomes. Further long-term randomized controlled trials are needed.

In conclusion, our data show that hemodynamic and biochemical parameters improved after the correction of HD patients' fluid imbalance, which was not detected clinically. Further prospective interventional trials are needed to evaluate the improvement of hard clinical endpoints with the change in volume status.

\section{Acknowledgments}

This study was supported in part by Fresenius Medical Care (FMC), Korea. However, FMC was not involved in the design of the study, collection, analysis or interpretation of the data, or the decision to submit the manuscript for publication. We have had no involvement from FMC that might raise a question of bias in the work reported or in the conclusions, implications or opinions stated. We thank all of the medical and nursing staff of the HD units at Seoul National University Hospital, Seoul National University Bundang Hospital and Gachon University Gil Hospital for their assistance. We also appreciate the statistical advice from the Medical Research Collaborating Centre (MRCC), Seoul National University Medical College.

\section{Conflict of Interest}

The authors declare no conflict of interest.

\section{References}

Bednarek-Skublewska, A., Zaluska, W. \& Ksiazek, A. (2010) The relationship between serum level of N-terminal pro-B-type natriuretic peptide and nutritional status, and inflammation in chronic hemodialysis patients. Clin. Nephrol., 73, 14-20.

Chamney, P.W., Wabel, P., Moissl, U.M., Muller, M.J., BosyWestphal, A., Korth, O. \& Fuller, N.J. (2007) A whole-body model to distinguish excess fluid from the hydration of major body tissues. Am. J. Clin. Nutr., 85, 80-89.

Cheng, L.T., Tang, W. \& Wang, T. (2005) Strong association between volume status and nutritional status in peritoneal dialysis patients. Am. J. Kidney Dis., 45, 891-902.

Ikeda, U., Ito, T. \& Shimada, K. (2001) Interleukin-6 and acute coronary syndrome. Clin. Cardiol., 24, 701-704.

Ikeda, U., Matsui, K., Murakami, Y. \& Shimada, K. (2002) Mono- 
cyte chemoattractant protein-1 and coronary artery disease. Clin. Cardiol., 25, 143-147.

Jacobs, L.H., van de Kerkhof, J.J., Mingels, A.M., Passos, V.L., Kleijnen, V.W., Mazairac, A.H., van der Sande, F.M., Wodzig, W.K., Konings, C.J., Leunissen, K.M., van Dieijen-Visser, M.P. \& Kooman, J.P. (2010) Inflammation, overhydration and cardiac biomarkers in haemodialysis patients: a longitudinal study. Nephrol. Dial. Transplant., 25, 243-248.

Jaeger, J.Q. \& Mehta, R.L. (1999) Assessment of dry weight in hemodialysis: an overview. J. Am. Soc. Nephrol., 10, 392-403.

Kotanko, P., Carter, M. \& Levin, N.W. (2006) Intestinal bacterial microflora - a potential source of chronic inflammation in patients with chronic kidney disease. Nephrol. Dial. Transplant., 21, 2057-2060.

Krack, A., Sharma, R., Figulla, H.R. \& Anker, S.D. (2005) The importance of the gastrointestinal system in the pathogenesis of heart failure. Eur. Heart J., 26, 2368-2374.

Machek, P., Jirka, T., Moissl, U., Chamney, P. \& Wabel, P. (2010) Guided optimization of fluid status in haemodialysis patients. Nephrol. Dial. Transplant., 25, 538-544.

McIntyre, C.W., Harrison, L.E., Eldehni, M.T., Jefferies, H.J., Szeto, C.C., John, S.G., Sigrist, M.K., Burton, J.O., Hothi, D., Korsheed, S., Owen, P.J., Lai, K.B. \& Li, P.K. (2011) Circulating endotoxemia: a novel factor in systemic inflammation and cardiovascular disease in chronic kidney disease. Clin. J. Am. Soc. Nephrol., 6, 133-141.

Mohamed-Ali, V., Goodrick, S., Bulmer, K., Holly, J.M., Yudkin, J.S. \& Coppack, S.W. (1999) Production of soluble tumor necrosis factor receptors by human subcutaneous adipose tissue in vivo. Am. J. Physiol., 277, E971-975.

Ouchi, N., Ohishi, M., Kihara, S., Funahashi, T., Nakamura, T., Nagaretani, H., Kumada, M., Ohashi, K., Okamoto, Y.,
Nishizawa, H., Kishida, K., Maeda, N., Nagasawa, A., Kobayashi, H., Hiraoka, H., Komai, N., Kaibe, M., Rakugi, H., Ogihara, T. \& Matsuzawa, Y. (2003) Association of hypoadiponectinemia with impaired vasoreactivity. Hypertension, 42, 231-234.

Passauer, J., Bussemaker, E. \& Gross, P. (1998) Dialysis hypotension: do we see light at the end of the tunnel? Nephrol. Dial. Transplant., 13, 3024-3029.

Passauer, J., Petrov, H., Schleser, A., Leicht, J. \& Pucalka, K. (2010) Evaluation of clinical dry weight assessment in haemodialysis patients using bioimpedance spectroscopy: a cross-sectional study. Nephrol. Dial. Transplant., 25, 545-551.

Pecoits-Filho, R., Barany, P., Lindholm, B., Heimburger, O. \& Stenvinkel, P. (2002) Interleukin-6 is an independent predictor of mortality in patients starting dialysis treatment. Nephrol. Dial. Transplant., 17, 1684-1688.

Shatat, I.F., Freeman, K.D., Vuguin, P.M., Dimartino-Nardi, J.R. \& Flynn, J.T. (2009) Relationship between adiponectin and ambulatory blood pressure in obese adolescents. Pediatr. Res., 65, 691-695.

Tattersall, J. (2009) Bioimpedance analysis in dialysis: state of the art and what we can expect. Blood Purif., 27, 70-74.

Vicente-Martinez, M., Martinez-Ramirez, L., Munoz, R., Avila, M., Ventura, M.D., Rodriguez, E., Amato, D. \& Paniagua, R. (2004) Inflammation in patients on peritoneal dialysis is associated with increased extracellular fluid volume. Arch. Med. Res., 35, 220-224.

Wizemann, V., Wabel, P., Chamney, P., Zaluska, W., Moissl, U., Rode, C., Malecka-Masalska, T. \& Marcelli, D. (2009) The mortality risk of overhydration in haemodialysis patients. Nephrol. Dial. Transplant., 24, 1574-1579. 